\title{
Tapered plasma channels to phase-lock accelerating and focusing forces in laser-plasma accelerators
}

\author{
W. Rittershofer, ${ }^{1, \text { a) }}$ C. B. Schroeder, ${ }^{2}$ E. Esarey, ${ }^{2}$ F. J. Grüner, ${ }^{1,}$ b) and W. P. Leemans ${ }^{2}$ \\ 1) Department für Physik, Ludwig-Maximilians-Universität München, Am Coulombwall 1, 85748 Garching, \\ Germany \\ ${ }^{2)}$ Lawrence Berkeley National Laboratory, Berkeley, California 94720 USA
}

(Dated: 16 May 2010)

Tapered plasma channels are considered for controlling dephasing of a beam with respect to a plasma wave driven by a weakly-relativistic, short-pulse laser. Tapering allows for enhanced energy gain in a single laserplasma accelerator stage. Expressions are derived for the taper, or longitudinal plasma density variation, required to maintain a beam at a constant phase in the longitudinal and/or transverse fields of the plasma wave. In a plasma channel, the phase velocities of the longitudinal and transverse fields differ, and, hence, the required tapering differs. The length over which the tapered plasma density becomes singular is calculated. Linear plasma tapering as well as discontinuous plasma tapering, which moves beams to adjacent plasma wave buckets, are also considered. The energy gain of an accelerated electron in a tapered laser-plasma accelerator is calculated and the laser pulse length to optimize the energy gain is determined.

PACS numbers: $52.38 . \mathrm{Kd}$

\section{INTRODUCTION}

Laser-plasma accelerators ${ }^{1}$ are of great interest because of their ability to sustain extremely large acceleration gradients, enabling compact accelerating structures. Laser-plasma acceleration is realized by using a high-intensity laser to ponderomotively drive a large plasma wave (or wakefield) in an underdense plasma. The plasma wave has relativistic phase velocity, approximately the group velocity of the laser, and can support large electric fields in the direction of laser propagation. When the laser pulse is approximately resonant (duration on the order of the plasma period) and the laser intensity is relativistic, with normalized laser vector potential $a=e A / m_{e} c^{2} \sim 1$, the size of the accelerating field is on the order of $E_{0}=c m_{e} \omega_{p} / e$, or $E_{0}[\mathrm{~V} / \mathrm{m}] \simeq 96 \sqrt{n_{0}\left[\mathrm{~cm}^{-3}\right]}$, where $\omega_{p}=\left(4 \pi n_{0} e^{2} / m_{e}\right)^{1 / 2}$ is the electron plasma frequency, $n_{0}$ is the ambient electron number density, $m_{e}$ and $e$ are the electron rest mass and charge, respectively, and $c$ is the speed of light in vacuum. In addition to extremely large accelerating gradients, plasma-based accelerators have the potential to produce extremely short electron bunches, a fraction of the plasma wavelength $\lambda_{p}=2 \pi c / \omega_{p}=2 \pi / k_{p}$. Therefore, laser-plasma accelerators are actively being investigated as ultra-compact sources of high-brightness beams for the next generation of light sources ${ }^{2-4}$ and linear colliders. ${ }^{5,6}$

High-quality electron beams up to $1 \mathrm{GeV}$ have been experimentally demonstrated in cm-scale plasma channels. ${ }^{7-9}$ The energy gain of a beam in the laser-driven plasma wave can be limited by several laser-plasma

a) Presently at University of Oxford, Clarendon Laboratory, Parks Road, Oxford OX1 3PU, UK.

b) Also at Max-Plank-Institut für Quantenoptik, HansKopfermann-Strasse 1, 85748 Garching, Germany. and beam-plasma interaction lengths. For typical laserplasma parameters, diffraction is the most severe limitation, and limits the interaction length to on the order of a few laser Rayleigh ranges. This limitation can be overcome through a combination of preformed plasma channel guiding, relativistic self-focusing, and ponderomotive self-channeling. ${ }^{10}$ In the regime of weakly-relativistic intensities $\left(a^{2} \lesssim 1\right)$ and moderate laser powers $P \lesssim P_{c}$, where $P_{c}$ is the critical power for relativistic self-focusing, a plasma channel alone can be employed to guide the laser over many Rayleigh ranges. Guiding at relativistic intensities over many Rayleigh ranges using preformed plasma channels has been demonstrated experimentally. ${ }^{11}$

With diffraction overcome by laser guiding, the beam energy can be limited by slippage between the beam position and the phase of the plasma wave. Consider an electron accelerated along the laser-propagation axis by a laser-driven plasma wave. In the linear regime, the field of the electrostatic plasma wave has the form $E_{z}=E_{\max } \sin \left[\omega_{p}\left(z / v_{p}-t\right)\right]$, where $v_{p}$ is the phase velocity of the plasma wave. As the electron is accelerated, its velocity will increase and approach the speed of light, $v_{z} \rightarrow c$. If the phase velocity of the plasma wave is constant with $v_{p}<c$, the electrons will eventually outrun the plasma wave and move into a phase region of the plasma wave that is decelerating. This limits the energy gain of the electron in the plasma wave and is commonly referred to as electron dephasing. The one-dimensional (1D) linear dephasing length $L_{d}$ can be defined as the length the electron must travel before it phase slips by one-half of a period with respect to the plasma wave. For a highly relativistic electron, $v_{z} \simeq c$, the linear dephasing length $L_{d}$ is given by $\left(1-v_{p} / c\right) L_{d}=\lambda_{p} / 2$, i.e., $L_{d} \simeq \gamma_{p}^{2} \lambda_{p}$, where $\gamma_{p}^{2}=\left[1-\left(v_{p} / c\right)^{2}\right]^{-1}$ and assuming $\gamma_{p}^{2} \gg 1$. If the phase velocity of the wake is assumed to be the linear group velocity of the laser $\gamma_{p} \simeq \gamma_{g} \simeq \omega_{0} / \omega_{p}$, where $\omega_{0}=2 \pi c / \lambda_{0}$ is the frequency of the laser, then the 1D linear dephas- 


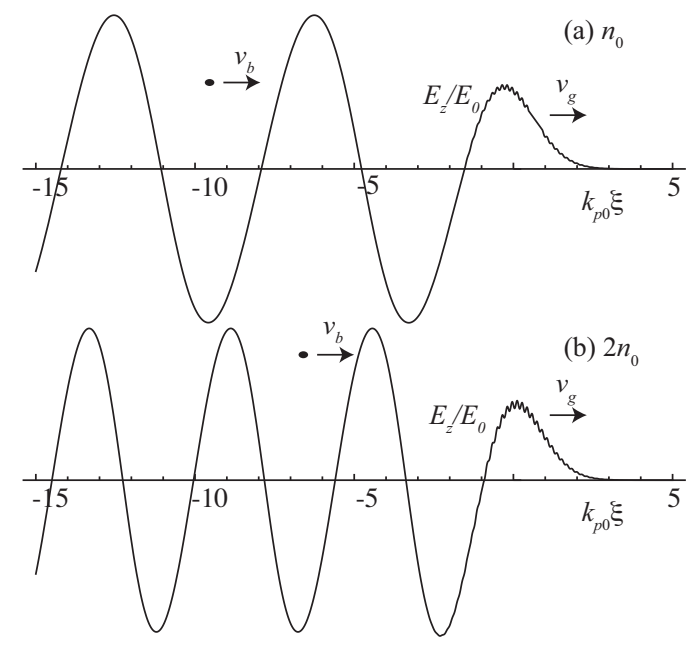

FIG. 1. Schematic of the basic principle of increasing the plasma density (tapering) to compensate for slippage between the plasma wave and a relativistic particle such that the particle (represented by a circle) remains in a constant phase of the plasma wave. (a) Longitudinal electric field $E_{z} / E_{0}$ at the initial density $n_{0}$. (b) Longitudinal electric field $E_{z} / E_{0}$ at the twice the initial density $2 n_{0}$. Laser centroid is located at $\xi=0$.

ing length is $L_{d 1 \mathrm{D}} \simeq \lambda_{p}^{3} / \lambda_{0}^{2}$. The maximum energy gain after a linear dephasing length is given approximately by $W_{\max } \approx e E_{\max } L_{d 1 \mathrm{D}}=2 \pi \gamma_{p}^{2}\left(E_{\max } / E_{0}\right) m_{e} c^{2}$, assuming $E_{\max }<E_{0}$. A more careful calculation ${ }^{12}$ gives a maximum energy gain of $W_{\max } \simeq 4 \gamma_{p}^{2}\left(E_{\max } / E_{0}\right) m_{e} c^{2}$, for $\left(2 \gamma_{p}\right)^{-2} \ll\left(E_{\max } / E_{0}\right)^{2} \ll 2$.

In addition to dephasing, the energy gain may be limited by laser depletion. As the laser propagates in the plasma, its energy is transferred to the plasma wave, and the rate of energy transfer ultimately limits the laserplasma interaction length. The characteristic laser depletion length ${ }^{13}$ is given, for a Gaussian laser pulse profile, by $L_{p d} \simeq 17.4\left(k_{0}^{2} / k_{p}^{3}\right) / a^{2}$, for $a^{2}<1$. In the weaklyrelativistic regime $a^{2}<1, L_{p d} \gg L_{d}$, and the energy gain will be limited by dephasing, not laser depletion.

It has been proposed that the dephasing limitation may be overcome by spatially tailoring the plasma density, which is referred to as plasma tapering. ${ }^{14-17}$ Physically, as the particles slip forward with respect to the drive laser, the plasma density is increased, reducing the plasma wavelength and maintaining the phase of the particles in the plasma wave bucket (cf. Fig. 1). Although tapering maintains the particle location in the phase of the plasma wave, slippage between the particles (with velocity $v_{b} \simeq c$ ) and the drive laser (with group velocity $\left.v_{g} \simeq 1-\omega_{p}(z)^{2} /\left(2 \omega^{2}\right)<c\right]$ will continue and limit the length over which tapering is possible. Injecting particles several plasma periods behind the laser will extend the distance over which one can taper the plasma density. Appropriate tapering can mitigate dephasing such that the energy gain is limited by laser depletion.

Although the concept of tapering is well-known, ${ }^{14-17}$ an explicit expression for the density taper in multidimensions is absent in the literature. Previous work considered tapering for 1D plasma waves, ${ }^{14-16}$ or assumed laser propagation in a plasma channel and neglected the transverse density gradient in the plasma response. ${ }^{17}$ Here a general expression for the optimal plasma density taper to phase-lock the beam in a plasma wave generated by a laser pulse guided in a plasma channel is derived. An analytic expression for the length over which the tapered plasma density becomes singular (i.e., the length over which tapering is possible) is also derived.

The plasma tapering required for maintaining a constant phase in the longitudinal field (accelerating force) and/or the transverse fields (focusing force) of the plasma wave is also obtained. In a plasma channel the phase velocity of the longitudinal wake differs from the transverse wake, and therefore the taper required to maintain a constant phase in the longitudinal wake differs from the taper that maintains a constant phase in the transverse wake. Variation of both the plasma channel radius and axial density allows the particle phase position of both the longitudinal and transverse fields to be held constant.

The energy gain of a highly relativistic electron in the plasma wave is calculated for various taper configurations, including tapering to phase lock a relativistic particle in the wake, a linear tapering, and a tapering that includes a density step. A linear tapering is considered since this may be easier to realize experimentally than the ideal tapering required for exact phase locking. To avoid a tapering profile in which the density becomes too large, a scenario is considered in which ideal tapering is used until the electron has phase slipped by one period of the plasma wave at the initial density, at which point the density is reduced back to its initial density. This has the effect of passing the electron forward by one plasma wave bucket while holding the phase in the plasma bucket constant. The optimal laser pulse length to maximize the energy gain in a tapered plasma is also calculated and found to be shorter than that in an untapered plasma.

This paper is organized as follows. Section II presents a derivation of the plasma wave excitation by a laser pulse propagating in a tapered plasma channel. In Sec. III the required plasma density taper for phase locking an electron to the longitudinal and/or transverse field, and the additional energy gain for the case of optimal plasma tapering are calculated. As the laser pulse propagates into the higher density plasma, the resonant condition for plasma wave excitation is shifted. The pulse duration for maximal energy gain over the optimally tapered plasma is calculated in Sec. IV A. The effect of a linear density taper, and the use of a discontinuous, quasi-periodic plasma density profile are considered in Secs. IV B and $\mathrm{V}$, respectively. A summary and conclusions are offered in Sec. VI. 


\section{PLASMA WAVE EXCITATION IN A TAPERED PLASMA CHANNEL}

In this section plasma wave excitation by a laser propagating in a non-uniform density channel is considered. A parabolic plasma channel that is slowly varying in the laser propagation direction $z$ can be characterized by two physical quantities: the on-axis density $n_{\mathrm{ch}}(z)$ and the matched laser spot size of a low-power laser pulse $r_{0 m}$, i.e., $n_{0}(z, r)=n_{\text {ch }}+\Delta n_{c} r^{2} / r_{0 m}^{2}$, where $\Delta n_{c}=1 /\left(\pi r_{e} r_{0 m}^{2}\right)$ is the critical channel depth, ${ }^{18}$ with $r_{e}=e^{2} / m_{e} c^{2}$. For a tapered plasma channel it is convenient to introduce the channel radius $R_{\mathrm{ch}}(z)$ such that $n_{0}(z, r)=n_{\mathrm{ch}}\left[1+r^{2} / R_{\mathrm{ch}}^{2}\right]$. A constant matched Gaussian laser spot size implies $n_{\mathrm{ch}} / R_{\mathrm{ch}}^{2}=\Delta n_{c} / r_{0 m}^{2}=$ constant. Therefore, the channel radius must increase as the plasma density is increased (such that a constant channel depth $\Delta n_{c}$ is maintained). ${ }^{17} \mathrm{~A}$ discussion of laser pulse propagation in a tapered plasma channel is presented in Appendix A. The plasma wavenumber can be expressed as $k_{p}^{2}(z, r)=4 \pi n_{0} e^{2} / m_{e} c^{2}=k_{p 0}^{2}\left[1+r^{2} / R_{\text {ch }}^{2}\right]$, where $k_{p 0}^{2}(z)=4 \pi n_{0}(z, 0) / m_{e} c^{2}$ is given by the on-axis plasma density.

\section{A. Plasma response}

The cold plasma equations for the plasma density $n$, normalized electron momentum $\mathbf{u}=\mathbf{p} /\left(m_{e} c\right)$, the normalized electrostatic potential $\varphi=e \phi /\left(m_{e} c^{2}\right)$ and the vector potential $\mathbf{a}=e \mathbf{A} /\left(m_{e} c^{2}\right)$ are

$$
\begin{gathered}
\frac{\partial n}{\partial c t}+\nabla \cdot\left(\frac{n \mathbf{u}}{\gamma}\right)=0 \\
\frac{\partial}{\partial c t}(\mathbf{u}-\mathbf{a})=\nabla(\phi-\gamma) \\
\nabla^{2} \phi=k_{p 00}^{2}\left(\frac{n-n_{0}}{n_{00}}\right), \\
{\left[\nabla^{2}-\frac{\partial^{2}}{\partial(c t)^{2}}\right] \mathbf{a}=k_{p 00}^{2} \frac{n}{n_{00}} \frac{\mathbf{u}}{\gamma}+\nabla \frac{\partial \phi}{\partial c t}}
\end{gathered}
$$

where $\gamma=\left(1+u^{2}\right)^{1 / 2}$ is the relativistic factor, $n_{0}(z, r)$ is the equilibrium density profile, and $n_{00}=n_{0}(0,0)$ and $k_{p 00}^{2}=4 \pi n_{00} e^{2} /\left(m_{e} c^{2}\right)$ are the initial on-axis density and plasma wave number, respectively. Here it is assumed that $\nabla \times(\mathbf{u}-\mathbf{a})=0$, i.e., initially zero helicity, and the Coulomb gauge $\nabla \cdot \mathbf{a}=0$ is used.

In the following the weakly-relativistic regime $|\boldsymbol{u}|<1$ is considered, such that the equations may be treated perturbatively with respect to the normalized amplitude of the laser field $a_{L}^{2} \ll 1$. In addition a broad channel is considered and it is assumed that the variation in plasma density owing to the axial taper is slow compared to the plasma wavelength and channel radius such that $\left|\partial_{z} n_{0}\right| \ll\left|\partial_{r} n_{0}\right| \ll k_{p} n_{0}$. To second order with respect to the normalized amplitude of the laser field $a_{L} \ll 1$,
Eqs. (1-4) become

$$
\begin{gathered}
\frac{\partial n_{2}}{\partial c t}+\nabla \cdot\left(n_{0} \mathbf{u}_{2}\right)=0 \\
\frac{\partial}{\partial c t}\left(\mathbf{u}_{2}-\mathbf{a}_{2}\right)=\nabla\left(\phi_{2}-a_{L}^{2} / 2\right), \\
\nabla^{2} \phi_{2}=k_{p 0}^{2}\left(\frac{n_{2}}{n_{00}}\right), \\
{\left[\nabla^{2}-\frac{\partial^{2}}{\partial(c t)^{2}}\right] \mathbf{a}_{2}=k_{p 0}^{2} \frac{n_{0}}{n_{00}} \mathbf{u}_{2}+\nabla \frac{\partial \phi_{2}}{c \partial t},}
\end{gathered}
$$

with $\nabla \cdot \mathbf{a}_{2}=0$, which can be combined to yield

$$
\left[\frac{\partial^{2}}{\partial(c t)^{2}}+k_{p}^{2}(z, r)+\nabla \times \nabla \times\right] \mathbf{u}_{2}=-\frac{\partial}{\partial c t} \nabla \frac{a_{L}^{2}}{2} .
$$

If the longitudinal taper is sufficiently slow, then a quasi-static approximation can be considered such that the laser evolution during propagation over a pulse duration (typically of the order of the plasma period $\sim \omega_{p}^{-1}$ ) may be neglected, i.e., $\partial_{z} \simeq-\partial_{c t}$. Here an axisymmetric geometry is assumed. The radial and axial components of Eq. (9) can then be combined to yield ${ }^{19}$

$$
\begin{aligned}
{\left[\frac{\partial^{2}}{\partial(c t)^{2}}+k_{p}^{2}-\nabla_{\perp}^{2}-\frac{1}{r} \frac{\partial}{\partial r} \frac{r}{k_{p}^{2}} \frac{\partial}{\partial r} \frac{\partial^{2}}{\partial(c t)^{2}}\right] \Psi } \\
=\left(k_{p}^{2}-\nabla_{\perp}^{2}\right) a_{L}^{2} / 2
\end{aligned}
$$

where $\Psi=\phi_{2}-a_{2 z}=u_{2 z}-a_{L}^{2} / 2$ is the effective potential. The longitudinal and transverse forces on a relativistic particle $v_{b} \simeq c$ are given by

$$
\begin{aligned}
E_{z} / E_{00} & =k_{p 00}^{-1} \partial_{c t} \Psi \\
\left(E_{r}-B_{\theta}\right) / E_{00} & =-k_{p 00}^{-1} \partial_{r} \Psi,
\end{aligned}
$$

where $E_{00}=m c^{2} k_{p 00} / e$.

To the lowest order in the normalized transverse scale length $\mathcal{O}\left(1 / k_{p} R_{\mathrm{ch}}\right)$, assuming a broad channel $k_{p} R_{\mathrm{ch}} \gg$ 1 , Eq. (10) is $\left(\partial_{c t}^{2}+k_{p}^{2}\right) \Psi_{0}=k_{p}^{2} a_{L}^{2} / 2$. Assuming that the laser field is primarily a function of the co-moving variable $a_{L}=a_{L}(\zeta)$ (where $\zeta$ is the distance relative to the laser centroid), the solution for $\Psi_{0}$ is

$$
\Psi_{0}=k_{p} \int_{\infty}^{\xi} \mathrm{d} \xi^{\prime} \sin \left[k_{p}\left(\xi-\xi^{\prime}\right)\right] a^{2}\left(\xi^{\prime}\right) / 2,
$$

where the variable co-moving with the laser centroid is

$$
\xi=\int_{0}^{z} d z^{\prime} / \beta_{g}\left(z^{\prime}\right)-c t
$$

Here $\beta_{g}$ is the linear $\left(a_{L}^{2} \ll 1\right)$ laser group velocity in a plasma channel

$$
\beta_{g}(z)=\left[1-4 /\left(r_{s} k_{0}\right)^{2}-k_{p 0}^{2} / k_{0}^{2}\right]^{1 / 2} .
$$

For an initially quiescent plasma ahead of the laser, the solution to Eq. (13) behind the laser driver takes the form

$$
\Psi_{0}=-\hat{A}(r) \sin \left[k_{p}(z, r) \xi+\varphi\right],
$$


where $\hat{A}$ is the amplitude of the plasma wave and $\varphi$ is a constant phase determined by the location of the drive laser. For a Gaussian laser pulse centered at $\xi=0, \varphi=0$ and $\hat{A}(r)=\hat{A}_{0} k_{p} L \exp \left(-2 r^{2} / r_{s}^{2}-k_{p}^{2} L^{2} / 2\right)$ behind the laser, where $r_{s} / 2$ is the rms waist of the laser intensity and $L$ is the rms length of the laser intensity, i.e., $a_{L}^{2} \propto$ $\exp \left(-\xi^{2} / 2 L^{2}\right)$.

Substituting Eq. (16) into Eqs. (11) and (12) yields ${ }^{19}$

$$
\begin{gathered}
\frac{E_{z}}{E_{00}}=\frac{k_{p} \hat{A}}{k_{p 00}} \cos \left(k_{p} \xi+\varphi\right) \\
\frac{\left(E_{r}-B_{\theta}\right)}{E_{00}}=\frac{\partial_{r} \hat{A}}{k_{p 00}} \sqrt{1+\left(\frac{\hat{A} \xi \partial_{r} k_{p}}{\partial_{r} \hat{A}}\right)^{2}} \sin \left(k_{p} \xi+\theta+\varphi\right),
\end{gathered}
$$

where $\tan (\theta)=\hat{A} \xi \partial_{r} k_{p} / \partial_{r} \hat{A}$.

In the following a laser nearly matched in a plasma channel with a broad laser spot $k_{p} r_{s} \gg 1$ is considered and terms of order $\mathcal{O}\left(k_{p} r_{s}\right)^{-2}$ are retained. The laser dynamics in a tapered channel is discussed in Appendix A. For a matched laser, the channel radius is given by $k_{p} R_{\text {ch }}=\left(k_{p} r_{s}\right)^{2} / 2$, and, hence, $\left(k_{p} R_{\mathrm{ch}}\right)^{2} \gg\left(k_{p} r_{s}\right)^{2} \gg 1$ for a broad channel. To lowest order for a Gaussian laser pulse in a parabolic channel, $\tan (\theta)=-k_{p 0} \xi r_{s}^{2} / 2 R_{\text {ch }}^{2}$. In this regime, the additional phase shift between the accelerating and focusing forces owing to the plasma channel will be small $\theta \ll 1$ for phases sufficiently close to the laser driver, i.e., provided $\left|k_{p} \xi\right|<k_{p} R_{\text {ch }}$.

\section{B. Linear wave phase velocity}

In a tapered plasma channel, the phase velocities of the axial $\left(E_{z}\right)$ and transverse $\left(E_{r}-B_{\theta}\right)$ wakefields are different, as described by Eqs. (17) and (18), owing to the $\theta$ term in the phase. Consider a particle near the channel axis $r \ll r_{s}$. The phases of the axial and radial forces experienced by the charged particle, i.e., $F_{z} \propto E_{z} \propto$ $\exp \left(i \psi_{z}\right)$ and $F_{r} \propto\left(E_{r}-B_{\theta}\right) \propto \exp \left(i \psi_{r}\right)$, respectively, can be expressed as

$$
\begin{aligned}
& \psi_{z}=k_{p 0} \xi \\
& \psi_{r}=k_{p 0} \xi\left(1-\frac{r_{s}^{2}}{2 R_{\mathrm{ch}}^{2}}\right)+\frac{\pi}{2}
\end{aligned}
$$

In general, the force (longitudinal or transverse) on the particle is a function $\propto \exp (i \psi)$ with a phase of the form $\psi=k_{p}(z) \xi f(z)$. The phase velocity of the field is $\beta_{p}=-\partial_{t} \psi / \partial_{z} \psi$, or

$$
\beta_{p}(z)=\left[\xi\left(k_{p 0} f\right)^{-1} \partial_{z}\left(k_{p 0} f\right)+1 / \beta_{g}(z)\right]^{-1} .
$$

In a tapered plasma, the phase velocity of the longitudinal field and the transverse fields are not equal. For example, for a matched pulse $\left(r_{s}=r_{0}=\right.$ constant $)$ in a tapered plasma channel with $R_{\mathrm{ch}}=k_{p 0} r_{0}^{2} / 2$, the longitudinal and transverse wake phase velocities, respectively, are

$$
\begin{aligned}
& \beta_{p z}=1+\frac{k_{p 0}^{2}}{2 k_{0}^{2}}\left(1+\frac{4}{k_{p 0}^{2} r_{s}^{2}}\right)-\frac{\xi \partial_{z} k_{p 0}}{k_{p 0}} \\
& \beta_{p r}=1+\left(\frac{k_{p 0}^{2}}{2 k_{0}^{2}}-\frac{\xi \partial_{z} k_{p 0}}{k_{p 0}}\right)\left(1+\frac{4}{k_{p 0}^{2} r_{s}^{2}}\right)
\end{aligned}
$$

In an axially-uniform plasma channel (i.e., without taper), then, from Eq. (21), the wave phase velocity is equal to the laser group velocity $\beta_{p}=\beta_{g}$. In this case, the difference between the plasma wave phase velocity and a highly-relativistic beam velocity near the laser propagation axis is

$$
\beta_{b}-\beta_{p} \simeq \frac{k_{p 0}^{2}}{2 k_{0}^{2}}\left(1+\frac{4}{k_{p 0}^{2} r_{s}^{2}}\right)
$$

where $\beta_{b}=v_{b} / c$ and $\gamma_{b}=\left(1-\beta_{b}^{2}\right)^{-1 / 2} \gg\left(k_{0} / k_{p 0}\right) \gg 1$. The length to $\operatorname{slip} \Delta \psi=\pi / 2$ (i.e., through a quarterperiod of the plasma wave corresponding to the accelerating and focusing phase region in the broad channel limit) is

$$
L_{d}=\frac{\left(k_{0} / k_{p 0}\right)^{2}}{\left[1+4 /\left(k_{p 0} r_{0}\right)^{2}\right]} \lambda_{p} / 2 .
$$

In the $1 \mathrm{D}$ limit, the length to $\operatorname{sip} \Delta \psi=\pi$ (i.e., through the accelerating phase of the plasma wave bucket with length $\left.\lambda_{p} / 2\right)$ is $k_{p 0}^{3} L_{d 1 \mathrm{D}} / k_{0}^{2}=2 \pi$, or $L_{d 1 \mathrm{D}} \simeq\left(k_{0} / k_{p 0}\right)^{2} \lambda_{p}$.

If the particle is to remain at a constant plasma wave phase, $\psi_{0}=$ constant, over the length of the interaction, then the phase velocity must be equal to the beam velocity. Assuming that the beam is highly-relativistic such that $\gamma_{b}^{2} \gg \gamma_{g}^{2} \gg 1$, then the phase velocity of the plasma wave must satisfy $\beta_{p} \simeq \beta_{b} \simeq 1$.

In general, Eq. (21) with $\psi_{0}=k_{p 0}(z) \xi f(z)$, can be written as

$$
-\psi_{0}\left[\frac{\partial_{z}\left(k_{p 0} f\right)}{k_{p 0}^{2} f^{2}}\right]=\beta_{g}^{-1}-\beta_{p}^{-1} .
$$

Assuming $\beta_{p} \simeq \beta_{b} \simeq 1$ yields

$$
-\psi_{0}\left[\frac{\partial_{z}\left(k_{p 0} f\right)}{k_{p 0}^{2} f^{2}}\right] \simeq \frac{k_{p 0}^{2}}{2 k_{0}^{2}}+\frac{2}{\left(k_{0} r_{s}\right)^{2}}
$$

where $\psi_{0}$ is assumed to be a constant to eliminate slippage. For a pulse centered at $\xi=0, \psi_{0}<0$ is the plasma wave phase location of the particles behind the laser pulse. For the phase of the longitudinal field given by Eq. (19), $f=1$, and for the phase of the transverse field given by Eq. $(20), f(z)=1-r_{s}^{2}(z) / 2 R_{\mathrm{ch}}^{2}(z)$. Equation (27) can be solved for the plasma density variation (taper) to eliminate slippage between the beam and the plasma wave fields. 


\section{PLASMA DENSITY TAPER}

In this section the longitudinal plasma density profile (taper) required to control the slippage between a relativistic beam and the phase of the longitudinal and/or transverse field of the plasma wave is calculated.

\section{A. Taper for longitudinal wake phase locking}

Consider a matched laser pulse propagating in a plasma channel such that $r_{s}(z)=r_{0}=$ constant. From Eq. (A3), the evolution of the channel radius must satisfy

$$
R_{\mathrm{ch}}(z)=k_{p 0}(z) r_{0}^{2} / 2
$$

Equation (28) indicates that, to maintain a matched laser spot, the channel radius must increase with the plasma density $R_{\mathrm{ch}}(z) \propto \sqrt{n_{0}(z)}$ such that the channel depth is constant $\Delta n_{c}=1 /\left(\pi r_{e} r_{0}^{2}\right)$.

The phase of the accelerating force is given by $\psi_{z}=$ $k_{p 0} \xi$. Equation (26) can be expressed in the case of the phase of the axial field (i.e., with $f=1$ ) as $^{17}$

$$
\frac{\mathrm{d} k_{p 0}}{\mathrm{~d} z}=\frac{k_{p 0}^{2}}{\left|\psi_{0}\right|}\left(\frac{1}{k_{0} Z_{R}}+\frac{k_{p 0}^{2}}{2 k_{0}^{2}}\right)
$$

where $Z_{R}=k_{0} r_{0}^{2} / 2$ is the Rayleigh length. Defining the normalized variables $\hat{k}_{p}=k_{p 0} / k_{p 00}, \hat{z}=k_{p 00}^{3} z / k_{0}^{2}$, and $\kappa=2 /\left(k_{p 00} r_{0}\right)$, Eq. (29) can be expressed as

$$
\frac{\mathrm{d} \hat{k}_{p}}{\mathrm{~d} \hat{z}}=\frac{\hat{k}_{p}^{2}}{2\left|\psi_{0}\right|}\left(\hat{k}_{p}^{2}+\kappa^{2}\right)
$$

with the general solution

$$
\begin{aligned}
& \hat{z}\left(\hat{k}_{p}\right)=\frac{2\left|\psi_{0}\right|}{\kappa^{2}}\left\{1-\hat{k}_{p}^{-1}\right. \\
& \left.\quad+\kappa^{-1} \arctan \left(\kappa^{-1}\right)\left[1-\frac{\arctan \left(\hat{k}_{p} \kappa^{-1}\right)}{\arctan \left(\kappa^{-1}\right)}\right]\right\} .
\end{aligned}
$$

The initial slope of the optimal taper is, from Eq. (30), $\mathrm{d} \hat{k}_{p} /\left.\mathrm{d} \hat{z}\right|_{\hat{z}=0}=\left(1+\kappa^{2}\right) /\left(2\left|\psi_{0}\right|\right)$, or

$$
\frac{\mathrm{d} n}{\mathrm{~d} z}(z=0)=\frac{n_{0}}{\left|\psi_{0}\right|} \frac{k_{p 00}^{3}}{k_{0}^{2}}\left(1+\frac{4}{k_{p 00}^{2} r_{0}^{2}}\right) .
$$

Equation (31) indicates that the density becomes singular at

$$
\hat{z}_{s}=\frac{2\left|\psi_{0}\right|}{\kappa^{2}}\left\{1-\kappa^{-1}\left[\pi / 2-\arctan \left(\kappa^{-1}\right)\right]\right\} .
$$

This singularity is the result of the slippage between the laser and the particle beam. As the plasma density is increased, the group velocity of the laser decreases, requiring a larger gradient in density to maintain a plasma wave phase velocity at the beam velocity. In the frame of the laser pulse, the beam is slipping forward with respect to the laser pulse. The singularity occurs once the beam reaches the laser, i.e., travels a distance $\left|\psi_{0}\right| / k_{p 00}$ with respect to the laser centroid. Note that the underdense plasma assumption will fail before the singular point is reached, and the model becomes invalid for high densities approaching $\hat{k}_{p}=k_{0} / k_{p 00} \gg 1$.

The maximum length of the plasma taper is given approximately by the length to reach the density singularity $L_{\text {taper }} \simeq z_{s}$. Using Eq. (33), $L_{\text {taper }} \simeq \hat{z}_{s} k_{p 00}^{3} / k_{0}^{2} \propto$ $n_{0}^{3 / 2}\left|\psi_{0}\right|$, where the phase $\left|\psi_{0}\right|=\left|k_{p 00} \xi(z=0, t=0)\right|$ is given by the initial distance of the particles behind the laser driver. Therefore, the length of the taper will be linearly proportional to the number of plasma wave buckets behind the laser driver where the beam is located, i.e., $L_{\text {taper }} \propto \hat{z}_{s} \propto\left|\psi_{0}\right| \propto N_{b}$, where $N_{b}$ is the number of plasma wave buckets behind the laser driver. The farther behind the laser driver, the longer the slippage distance (for the beam to reach the laser), and the longer the taper length. As shown in Sec. IV A, the maximum energy gain with taper is also linearly proportional to the number of plasma wave buckets behind the laser driver where the beam is initially located.

In deriving the phase of the plasma wave fields in Sec. II, a broad laser pulse $\left(k_{p} r_{s}\right)^{2} \gg 1$ was assumed. For a broad pulse, in the limit $\kappa^{2}=4 /\left(k_{p 0} r_{0}\right)^{2} \ll 1$, the plasma density taper Eq. (31) can be expressed as

$$
\hat{k}_{p}(\hat{z}) \simeq \frac{1-\left(1-3 \hat{z} / 2\left|\psi_{0}\right|\right)^{2 / 3} \kappa^{2} / 5}{\left(1-3 \kappa^{2} / 5-3 \hat{z} / 2\left|\psi_{0}\right|\right)^{1 / 3}}
$$

and the singular point (maximum taper length) to lowest order in $\kappa^{2} \ll 1$ is

$$
\hat{z}_{s}=\frac{2\left|\psi_{0}\right|}{3}\left(1-3 \kappa^{2} / 5\right) \text {. }
$$

The expressions for the optimal axial taper to the next order in the radial parameter [i.e., to order $\mathcal{O}\left(\kappa^{4}\right)$ ] are presented in Appendix B. The energy gain for a plasma taper given by Eq. (34) is calculated in Sec. IV. In the 1D limit, $\kappa \rightarrow 0$, and the plasma taper takes the form ${ }^{16}$ $\hat{k}_{p}(z)=\left(1-3 \hat{z} / 2\left|\psi_{0}\right|\right)^{-1 / 3}$, with the singular point $\hat{z}_{s}=$ $2\left|\psi_{0}\right| / 3$.

The optimal plasma density taper to lock the phase of the accelerating field is plotted in Fig. 2, which shows the taper required to maintain a constant accelerating phase $\left|\psi_{0}\right|=3 \pi$ for $\kappa=0$ and $\kappa=0.3$. The dashed curves are the approximate solution, for $\kappa^{2} \ll 1$, Eq. (34). As an example, for a laser with wavelength $0.8 \mu \mathrm{m}$ and matched spot $r_{0}=25 \mu \mathrm{m}$ propagating in a tapered plasma channel with initial on-axis density $2 \times 10^{18} \mathrm{~cm}^{-3}$, such that $2 \pi / k_{p 00} \simeq 30 \mu \mathrm{m}$ and $\kappa=2 /\left(k_{p 00} r_{0}\right)=0.3$, the tapered plasma density will increase to $8 \times 10^{18} \mathrm{~cm}^{-3}$ (to $\left.\hat{k}_{p}=2\right)$ after a distance of $z \simeq 1.7 \mathrm{~cm}(\hat{z}=5.2)$. 


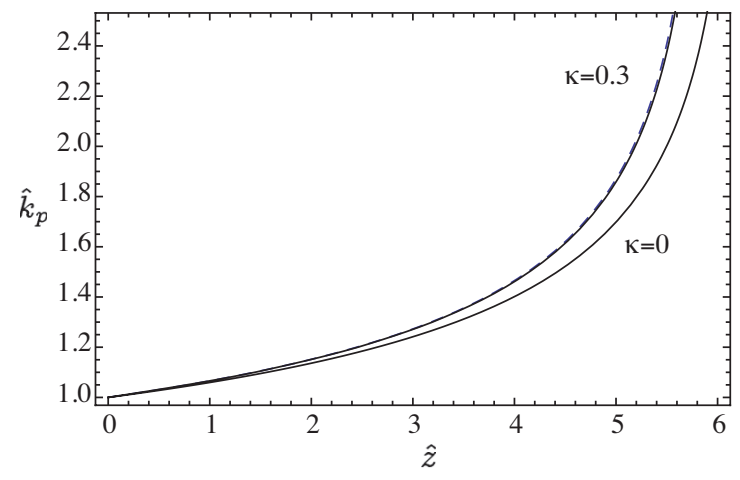

FIG. 2. The normalized plasma frequency $\hat{k}_{p}=k_{p 0}(z) / k_{p 00}$ versus $\hat{z}=k_{p 00}^{3} z / k_{0}^{2}$ for phase locking the accelerating force (constant phase $\left|\psi_{0}\right|=3 \pi$ ) for $\kappa=0$ and $\kappa=0.3$. The dashed curves are the approximate solution, Eq. (34).

\section{B. Taper for transverse wake phase locking}

The radially varying density in the plasma channel produces a phase shift between the longitudinal and transverse forces [cf. Eqs.(19) and (20)]. Hence, if the longitudinal phase is made constant by employing a density taper given by Eq. (34) a beam will slip with respect to the transverse fields. For a beam experiencing a linear focusing force, the matched electron beam radius is $\sigma_{r}=\left(\varepsilon_{\perp} / k_{\beta}\right)^{1 / 2}$, where $\varepsilon_{\perp}$ is the transverse geometric emittance and the betatron wavenumber $k_{\beta}$ is determined from the focusing force. In a high energy accelerator $\gamma \gg 1$, external focusing between accelerator sections will most likely be employed, and the plasma focusing should be made near zero. For a uniform plasma, this may be accomplished by making the laser spot sufficiently broad $k_{p} r_{s} \gg 1$, or shaping the transverse profile of the laser such that $\partial_{r} a^{2}=0$ near the axis. In a plasma channel, one can consider a combination of highorder laser modes to produce $\partial_{r} a^{2}=0,{ }^{20}$ or one can consider placing the beam at the zero crossing of the focusing field. In either case, the phase location of the beam in the transverse fields should remain fixed to minimize emittance growth. For a beam with constant transverse wake phase, the phase of the accelerating field slips with respect to the beam, resulting in a modest decrease in energy gain.

To lock the phase of the transverse field of a matched laser pulse requires $\psi_{r}=k_{p 0} f \xi=$ constant, with $f=$ $1-r_{0}^{2} /\left(2 R_{\mathrm{ch}}^{2}\right)=1-\kappa^{2} /\left(2 \hat{k}_{p}^{2}\right)$. From Eq. (26), the equation for optimal tapering to maintain the transverse field is

$$
\frac{\mathrm{d} \hat{k}_{p}}{\mathrm{~d} \hat{z}}=\frac{\hat{k}_{p}^{2}}{2\left|\psi_{0}\right|}\left(\hat{k}_{p}^{2}-\kappa^{2} / 2\right)
$$

for a broad pulse $\kappa^{2} \ll 1$. The solution to Eq. (36) is

$$
\hat{k}_{p}(\hat{z}) \simeq \frac{1-\left[1-3 \hat{z} /\left(2\left|\psi_{0}\right|\right)\right]^{2 / 3}\left(\kappa^{2} / 10\right)}{\left[1+3 \kappa^{2} / 10-3 \hat{z} /\left(2\left|\psi_{0}\right|\right)\right]^{1 / 3}} .
$$

Note that the beam interaction with the plasma wave will stop once the beam has slipped forward sufficiently far to reach the laser pulse. This distance is given by $\hat{z}_{s}$, Eq. (35), which occurs before the singularity in Eq. (37) is reached.

For the case where the phase of the transverse wake is held constant using a plasma density taper, with a matched laser $r_{s}=r_{0}$, the phase of the beam in the longitudinal wake is

$$
\psi_{z}=k_{p 0} \xi=\left(\psi_{r}+\pi / 2\right)\left(1+\kappa^{2} / 2 \hat{k}_{p}^{2}\right),
$$

where the transverse wake phase is now fixed $\psi_{r}=$ constant, and $\hat{k}_{p}(z)$ is given by equation Eq. (37). As the beam propagates the phase of the axial field increases.

\section{Taper for longitudinal and transverse wake phase locking}

The plasma density can be varied to lock the phase of the accelerating or the focusing fields for a matched laser propagating in a plasma channel driving a plasma wave, but not both. Matched laser propagation requires that the channel radius and on-axis plasma density satisfy Eq. (28). By allowing both the channel radius and the plasma density to differ from the matched condition, both the accelerating phase and focusing phase can be phase-locked to a beam. In this case the laser spot size will no longer be matched to the plasma channel. If the on-axis longitudinal field Eq. (19) is constant, then the transverse phase Eq. (20) will be constant provided $r_{s} / R_{\mathrm{ch}}=$ constant. Hence as the plasma density varies, the matched channel condition Eq. (28) can no longer be satisfied, causing the channel radius and laser spot size to decrease, and the laser intensity to increase: $a^{2}=a_{0}^{2}\left(r_{0} / r_{s}\right)^{2}$.

Maintaining constant axial and transverse phase (while allowing the laser spot size to vary) requires

$$
\frac{\mathrm{d} \hat{k}_{p}}{\mathrm{~d}\left(z / Z_{R}\right)}=\epsilon \hat{k}_{p}^{2}\left(\hat{k}_{p}^{2}+\frac{\kappa^{2}}{R^{2}}\right)
$$

where $R=r_{s}(z) / r_{0}$ and $\epsilon=k_{p 00}^{3} Z_{R} /\left(2\left|\psi_{0}\right| k_{0}^{2}\right)$ is the ratio of the laser spot evolution length scale to the plasma taper length scale. The laser spot-size evolution in a tapered plasma channel, given by Eq. (A3), can be expressed as

$$
\frac{R^{3} \mathrm{~d}^{2} R}{\mathrm{~d}\left(z / Z_{R}\right)^{2}}=1-\hat{k}_{p}^{2} R^{2}
$$

assuming $r_{s}(z) / R_{\mathrm{ch}}(z)=r_{0} / R_{\mathrm{ch}}(0)=$ constant and an initially matched laser $R_{\mathrm{ch}}(0)=k_{p 0}(0) r_{0}^{2} / 2$.

The coupled system, Eqs. (39) and (40), can be solved by assuming the taper is slow compared to the laser spot evolution and $\epsilon \ll 1$. Consider a perturbation about the matched laser spot $R=1+\epsilon R_{1}$ and axial plasma density 


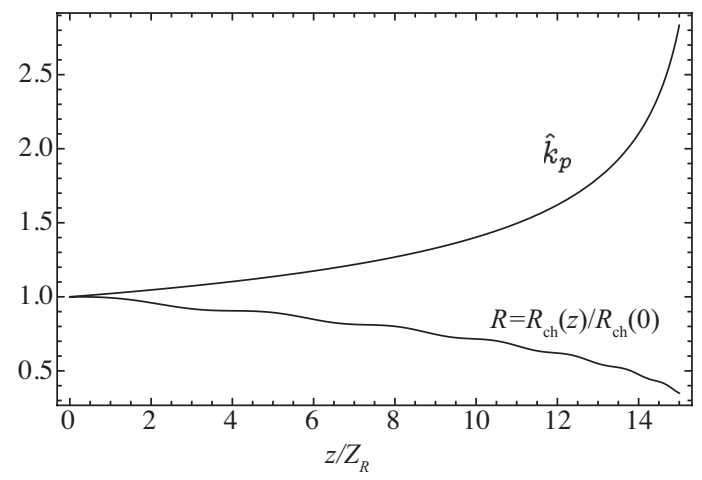

FIG. 3. Normalized laser spot size and channel radius $R=r_{s}(z) / r_{0}=R_{\mathrm{ch}}(z) / R_{\mathrm{ch}}(0)$ and plasma frequency $\hat{k}_{p}$ versus propagation distance (normalized to the Rayleigh range) $z / Z_{R}$ to maintain a constant phase in the accelerating and focusing phases for $\epsilon=0.02$ and $\kappa=0.25$.

$\hat{k}_{p}=1+\epsilon \hat{k}_{p 1}$. For $z \ll z_{s}$, the lowest order solutions to the coupled equations are

$$
\begin{aligned}
\hat{k}_{p}(z) & =1+\epsilon\left(1+\kappa^{2}\right)\left(z / Z_{R}\right), \\
R(z) & =1-2 \epsilon\left(1+\kappa^{2}\right)\left[\left(z / Z_{R}\right)-\sin \left(z / Z_{R}\right)\right] .
\end{aligned}
$$

The plasma density initially increases linearly and the spot size decreases (and oscillates with period $Z_{R} / 2 \pi$ ). Figure 3 shows the numerical solution to the coupled Eqs. (39) and (40) for the parameters $\epsilon=0.02$ and $\kappa=0.25$. For example, $\epsilon=0.02$ and $\kappa=0.25$ correspond to tapering to lock a particle at the phase $\psi_{0}=-5 \pi$ in a plasma wave driven by a $0.8 \mu \mathrm{m}$ wavelength laser with an initially matched $54 \mu \mathrm{m}$ spot $\left(Z_{R} \simeq 1.2 \mathrm{~cm}\right)$ propagating in a plasma channel with on-axis density $6 \times 10^{17} \mathrm{~cm}^{-3}$. In Fig. 3 the channel radius has decreased to $R_{\mathrm{ch}}(z)=0.5 R_{\mathrm{ch}}(0)$ over $z=13.8 Z_{R}$, and the laser spot has decreased to $r_{s}=27 \mu \mathrm{m}$, with an increase in laser intensity of a factor of 4 .

\section{ENERGY GAIN WITH TAPER}

Employing a tapered plasma channel, as described in Sec. III, yields increased energy gain in a single accelerator stage limited by dephasing (for the case of low depletion, e.g., for weakly-relativistic laser intensities $a \lesssim 1)$. The energy gain may be estimated by considering a charged particle propagating near the axis $r_{b} \ll r_{s}$. Assuming the laser pulse envelope to be Gaussian with rms pulse length $L$, the laser-period averaged envelope of the normalized intensity is $a^{2}=\left(a_{0}^{2} / 2 R^{2}\right) \exp \left(-\xi^{2} / 2 L^{2}\right)$. Applying Eqs. (11) and (13), the on-axis accelerating electric field behind the laser pulse is

$$
E_{z}=\frac{a_{0}^{2}}{R^{2}} \sqrt{\frac{\pi}{8}} \frac{m_{e} c^{2}}{e} k_{p 0}^{2} L \mathrm{e}^{-\left(k_{p 0} L\right)^{2} / 2} \cos \left(k_{p 0} \xi+\varphi\right) .
$$

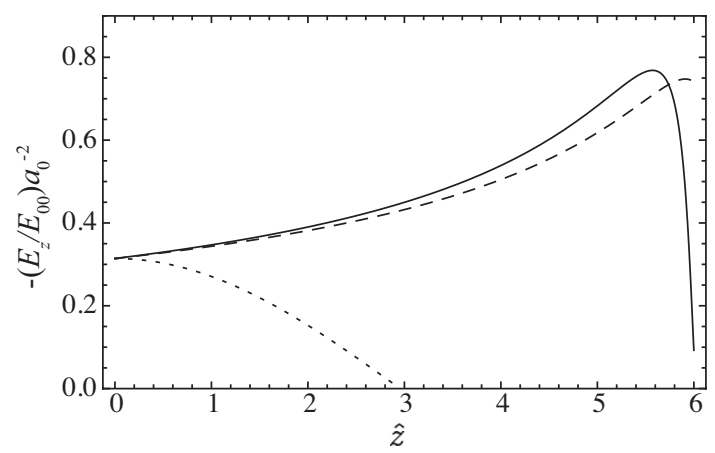

FIG. 4. Normalized plasma wave accelerating field at $(r, z, t)=\left(0, z, z / c \beta_{b}\right)$ with $\kappa=0.25$ and $\hat{L}=0.6$ versus propagation distance $\hat{z}$ for the cases: without taper (dotted curve), taper given by Eq. (34) (solid curve), and taper given by Eq. (37) (dashed curve). The initial phase of the particle is $\psi_{z}(0)=-3 \pi$.

For a relativistic particle $\gamma_{b}^{2} \gg \gamma_{g}^{2} \gg 1$, the energy gain can be expressed as

$$
\Delta \gamma=a_{0}^{2} \frac{k_{0}^{2}}{k_{p 00}^{2}} \sqrt{\frac{\pi}{8}} \hat{L} \int d \hat{z} \hat{k}_{p}^{2} \mathrm{e}^{-\hat{k}_{p}^{2} \hat{L}^{2} / 2} R^{-2} \cos \left(k_{p} \xi+\varphi\right),
$$

with the normalized pulse length $\hat{L}=k_{p 00} L$.

Without taper $\left(k_{p 0}=k_{p 00}\right)$ and assuming a matched $(R=1)$, resonant $(\hat{L}=1)$ laser pulse, the maximum energy gain for a highly-relativistic $\left(\beta_{b}=1\right)$ electron propagating near the channel axis $\left(r \ll r_{s}\right)$ is $\Delta \gamma=(\pi / 2 \mathrm{e})^{1 / 2}\left(1+\kappa^{2}\right)^{-1} a_{0}^{2}\left(k_{0} / k_{p 0}\right)^{2}$, over an acceleration length (i.e., linear dephasing length) of $\hat{L}_{d}=$ $k_{p 0} L_{d}=\pi\left(k_{0} / k_{p 0}\right)^{2}\left(1+\kappa^{2}\right)^{-1}$.

With a plasma taper, the phase of the accelerating field can be made constant, thereby increasing the energy gain. Figures 4 and 5 show the electric field Eq. (43) and the energy gain Eq. (44), respectively, assuming a matched laser $(R=1)$. The cases without taper (dotted curves), with a taper to lock the position of the particle in the longitudinal phase given by Eq. (34) (solid curves), and with a taper to lock the particle position in the transverse wake phase (dashed curves) are shown in Figs. 4 and 5 assuming $\hat{L}=0.6$ and $\psi_{z}(0)=-3 \pi$. As an example, for a laser with wavelength $0.8 \mu \mathrm{m}$, normalized intensity $a_{0}=0.4$, and matched spot $r_{0}=95 \mu \mathrm{m}$ propagating in a tapered plasma channel with initial onaxis density $2 \times 10^{17} \mathrm{~cm}^{-3}$, such that $2 \pi / k_{p 00} \simeq 75 \mu \mathrm{m}$ and $\kappa=2 /\left(k_{p 00} r_{0}\right)=0.25$, the initial peak electric field (Fig. 4) is $\left|E_{z}(z=0)\right| \simeq 3.1 E_{00} a_{0}^{2} \simeq 2.2 \mathrm{GV} / \mathrm{m}$. For these parameters the energy gain with tapering to lock the longitudinal wake (Fig. 5) is $m c^{2} \Delta \gamma \simeq 2.1 \mathrm{GeV}$ compared to $0.42 \mathrm{GeV}$ without taper. Note that self-focusing of the laser was neglected. Once the plasma density increases via tapering such that $P / P_{c}=\left(k_{p 0} r_{0} a_{0}\right)^{2} / 32>1$, relativistic self-focusing will result in laser evolution. 


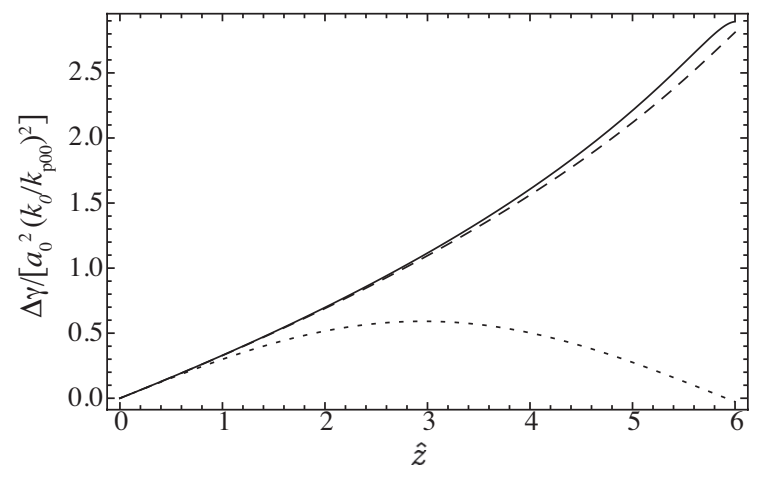

FIG. 5. Normalized energy gain $\Delta \gamma /\left[a_{0}^{2}\left(k_{0} / k_{p 00}\right)^{2}\right]$ with $\kappa=$ $0.25, \hat{L}=0.6$, and $\psi_{z}(0)=-3 \pi$ versus propagation distance $\hat{z}=k_{p 00}^{3} z / k_{0}^{2}$ for the cases: without taper (dotted curve), taper given by Eq. (34) (solid curve), and taper given by Eq. (37) (dashed curve).

\section{A. Maximum energy gain with longitudinal wake tapering}

For a matched pulse, and constant phase of the longitudinal wake, the energy gain Eq. (44) may be expressed as

$$
\Delta \gamma=a_{0}^{2} \sqrt{\frac{\pi}{8}} \frac{k_{0}^{2}}{k_{p 0}^{2}} \hat{L} \cos \left(\psi_{0}\right) \int_{1}^{\infty} d \hat{k}_{p} \frac{\hat{k}_{p}^{2} \mathrm{e}^{-\hat{k}_{p}^{2} \hat{L}^{2} / 2}}{\left(d \hat{k}_{p} / d \hat{z}\right)}
$$

where $d \hat{k}_{p} / d \hat{z}$ is given by Eq. (30) and integration is to the singular point $\hat{z}_{s}\left(\hat{k}_{p} \rightarrow \infty\right)$. Note that, although the underdense assumption of this theory breaks down as the plasma density approaches the critical density, the excited wake field goes to zero exponentially for $k_{p 0} L \gg 1$ and hence there are negligible differences between taking the upper limit of the integral of a few or infinity.

Assuming a broad pulse $\kappa^{2} \ll 1, \hat{k}_{p}^{2}\left(d \hat{z} / d \hat{k}_{p}\right) \simeq$ $\left(2\left|\psi_{0}\right| / \hat{k}_{p}^{2}\right)\left(1-\kappa^{2} / \hat{k}_{p}^{2}\right)$, Eq. (45) can be integrated with the solution

$$
\Delta \gamma=a_{0}^{2}\left(\frac{k_{0}}{k_{p 0}}\right)^{2} \sqrt{\frac{\pi}{8}}\left(2\left|\psi_{0}\right|\right) \cos \left(\psi_{0}\right) \Lambda(\hat{L}),
$$

where

$$
\begin{aligned}
\Lambda(\hat{L})=\hat{L} \mathrm{e}^{-\hat{L}^{2} / 2}\left[1-\frac{\kappa^{2}}{3}\left(1-\hat{L}^{2}\right)\right] \\
-\sqrt{\frac{\pi}{2}} \hat{L}^{2}\left(1+\frac{\kappa^{2}}{3} \hat{L}^{2}\right) \operatorname{Erfc}(\hat{L} / \sqrt{2}) .
\end{aligned}
$$

Figure 6 shows the energy gain versus laser pulse duration. The optimal initial laser pulse duration for maximum energy gain at can be found by solving $d \Lambda / d \hat{L}=0$, which yields $\hat{L} \simeq 0.612-0.085 \kappa^{2}$ for $\kappa \ll 1$.

\section{B. Linear plasma density taper}

A plasma channel with optimal longitudinal density tapering Eq. (31) may be challenging to realize experi-

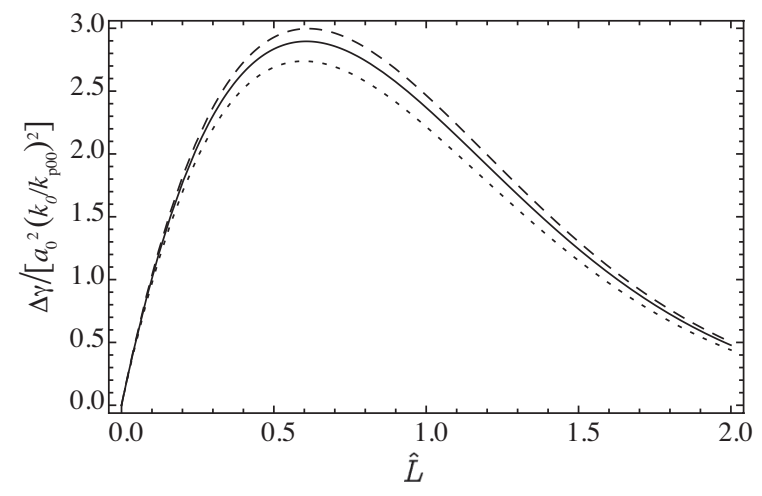

FIG. 6. Normalized energy gain $\Delta \gamma /\left[a_{0}^{2}\left(k_{0} / k_{p 00}\right)^{2}\right]$ versus normalized initial laser pulse duration $\hat{L}=k_{p 00} L$ for $\kappa=0$ (dashed curve), $\kappa=0.25$ (solid curve), and $\kappa=0.4$ (dotted curve). $\hat{L}=1$ corresponds to an initially, linearly resonant laser pulse.

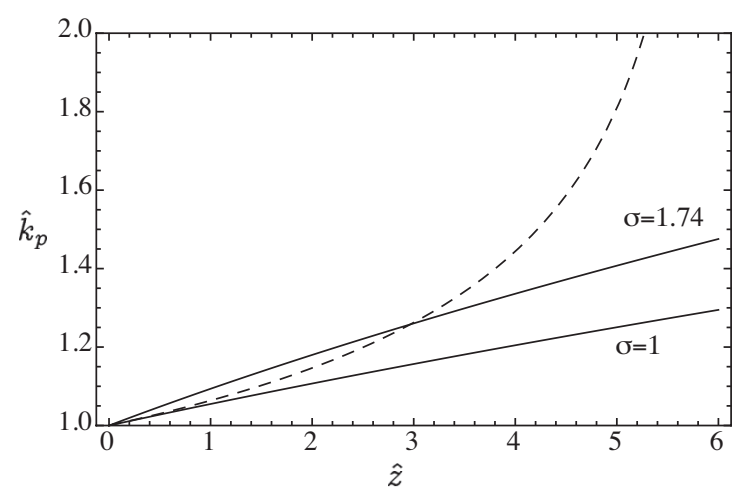

FIG. 7. The normalized plasma frequency $\hat{k}_{p}=k_{p 0}(z) / k_{p 00}$ versus $\hat{z}=k_{p 00}^{3} z / k_{0}^{2}$ with initial phase $\psi_{0}=-3 \pi$ and $\kappa=$ 0.25 , for $\sigma=1, \sigma=1.74$, and the optimal taper (dashed curve) given by Eq. (34).

mentally. In this section a linear plasma density taper, which may be simpler to realize in practice, is considered and shown to also significantly increase the energy gain.

The plasma density can be expressed in terms of the initial density slope for the case of optimal tapering,

$$
\hat{k}_{p}^{2}(\hat{z})=\frac{n(\hat{z})}{n_{00}}=1+2 \sigma \frac{d \hat{k}_{p}(0)}{d \hat{z}} \hat{z}
$$

where $d \hat{k}_{p}(\hat{z}=0) / d \hat{z}=\left(1+\kappa^{2}\right) /\left(2\left|\psi_{0}\right|\right)$, is the initial slope of the plasma wavenumber and $\sigma$ is a constant that can be determined to yield the maximum energy gain. Figure 7 shows the plasma density taper (with initial phase $\psi_{0}=-3 \pi$ and $\left.\kappa=0.25\right)$ for $\sigma=1, \sigma=1.74$, and the optimal taper given by Eq. (34). For densities less than the optimal taper, the $\beta_{b} \simeq 1$ beam slips forward with respect to the longitudinal wake, and for densities larger than the optimal taper the $\beta_{b} \simeq 1$ beam slips back with respect to the longitudinal wake.

Assuming the initial phase of the particle is $\psi_{0}$, in a linear taper the phase of the particle slips with respect 


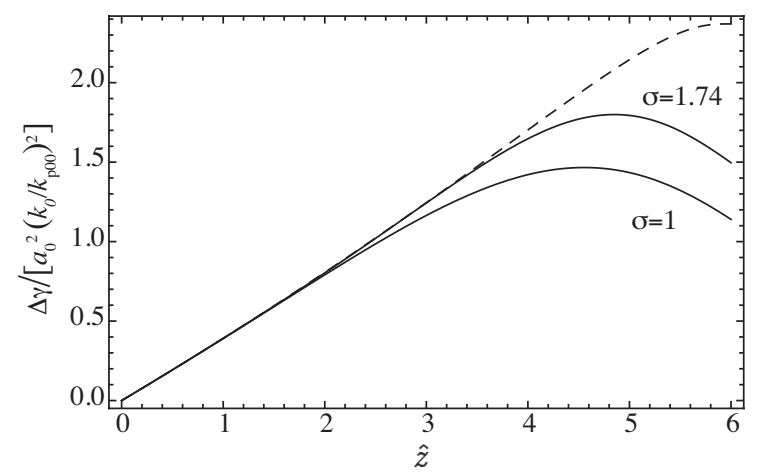

FIG. 8. Normalized energy gain for linear tapers with $\kappa=$ 0.25 and $\psi_{0}=-3 \pi$ for $\sigma=1$ and $\sigma=1.74$ versus propagation distance $\hat{z}$. Dashed curve is energy gain with optimal taper.

to the axial field such that

$$
\psi_{z}=\hat{k}_{p}\left(\psi_{0}+\hat{z}\left[1+\sigma \hat{z}\left(d \hat{k}_{p}(0) / d \hat{z}\right)\right]\left(1+\kappa^{2}\right) / 2\right),
$$

where terms of order $\mathcal{O}\left(k_{p 00}^{2} / k_{0}^{2}\right)$ are retained. Figure 8 shows the energy gain by solving Eq. (44), with $\hat{L}=1$, $\kappa=0.25$, and injection at $\psi_{0}=-3 \pi$, for the three tapers shown in Fig. (7). For the case of $\sigma=1.74$ the beam initially slips back with respect to the longitudinal wake, and then slips forward before moving into a defocusing region of the plasma wave.

\section{STEP PLASMA DENSITY TAPER}

The length over which tapering (to phase-lock a particle in the plasma wave) is possible is limited by the density growing unbounded near $\hat{z}_{s}$. Tapering may be extended by injecting the beam into a plasma wave phase further behind the laser (i.e., increasing $\left|\psi_{0}\right|$ ) or by considering a discontinuous step in the plasma density profile. For example, consider tapering to maintain the phase of the beam in the plasma wave. After the beam slips, with respect to the laser, one plasma wavelength of the initial density, then (instantaneously) reducing the density to the initial value will keep the beam in the same phase of the plasma wave, however, in one plasma wave bucket closer to the laser compared to its initial position. A discontinuous or layered taper was considered in Ref. 16.

The distance to slip $N_{s}$ periods of the plasma wave with respect to the initial plasma density is given by $k_{p 00} \xi=\psi_{0}+2 \pi N_{s}$, where $\psi_{0}$ is the initial phase that is held constant by the tapering, $k_{p 0} \xi=\hat{k}_{p}\left(k_{p 00} \xi\right)=\psi_{0}$. Therefore, the beam will have slipped $N_{s}$ periods with respect to the initial plasma density after the tapering has increased the density to $\hat{k}_{p}=\psi_{0} /\left(\psi_{0}+2 \pi N_{s}\right)$. The distance to reach this density taper is given by Eq. (31). In this process, the beam has moved from $N_{b}$ plasma wave buckets behind the laser to $N_{b}-N_{s}$ buckets behind the

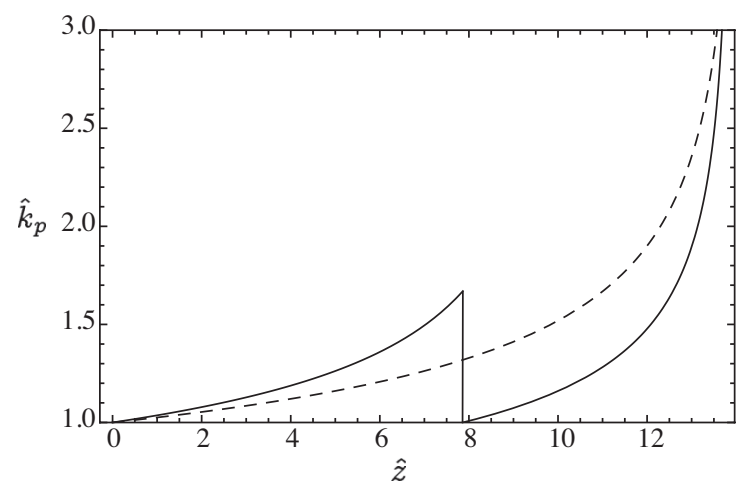

FIG. 9. Normalized plasma wavenumber $\hat{k}_{p}$ versus distance $\hat{z}$ with $\kappa=0.25$. Solid curve is the density taper for a beam that is in the initial phase $\psi_{0}=-5 \pi$ and moves to the phase $\psi_{1}=-3 \pi$ after the density discontinuity at $\hat{z}\left(\hat{k}_{p}=5 / 3\right)$. Dashed curve is the continuous density taper for a beam at the phase $\psi_{0}=-7 \pi$.

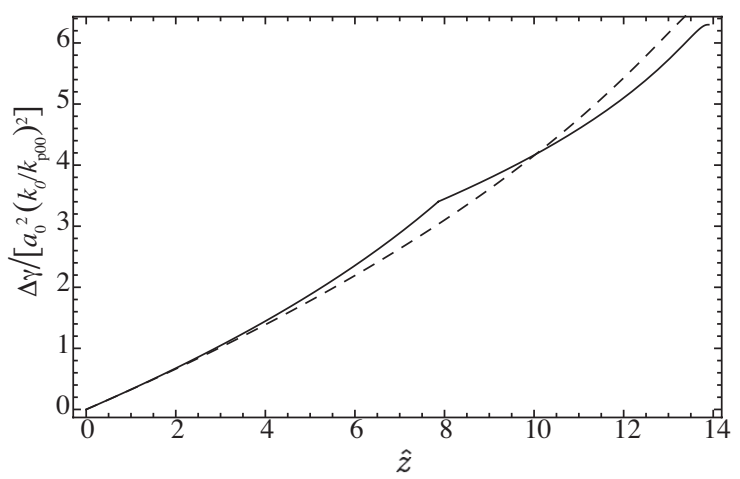

FIG. 10. Normalized energy gain with $\hat{L}=0.6$ for the taper cases shown in Fig. 9: solid curve is the discontinuous taper case and dashed curve is the continuous taper case.

laser after the density discontinuity, while maintaining the phase in the plasma wave. In principle, the stepping down in density described here can be repeated, advancing the beam to plasma wave buckets closer to the laser pulse, until the beam reaches the first plasma wave bucket.

Figure 9 shows an example (with $\kappa=0.25$ ) of a plasma density tapering where the beam is initially at the phase $\psi_{0}=-5 \pi$, and after propagating a distance such that $\hat{k}_{p}=5 / 3$, the density is reduced to the initial value at $\hat{z}\left(\hat{k}_{p}=5 / 3\right) \simeq 7.86$, and the beam is then located at the phase $\psi_{1}=-3 \pi$. The plasma may then be tapered via Eq. (34) to maintain this phase, as shown in Fig. 9. Effectively, the beam is starting in the third plasma wave bucket behind the laser, and after the density discontinuity, moves to the second plasma wave bucket behind the laser pulse. For comparison, also shown in Fig. 9 is the continuous taper for a beam initially at a phase $\psi_{0}=-7 \pi$ (dashed curve), i.e., a beam initially in the fourth plasma wave bucket behind the laser pulse.

Figure 10 shows the energy gain for the two cases (dis- 
continuous taper and continuous taper) shown in Fig. 9. Although the energy gains are comparable, Fig. 10 shows that higher energy gain can be achieved over equal propagation distance by simply using a continuous taper and injecting at a phase further being the laser (dashed curve), compared to discontinuous tapering (solid curve).

\section{SUMMARY AND CONCLUSIONS}

In a longitudinally homogeneous plasma, the beam velocity and the laser-driven plasma wave phase velocity are not matched, resulting in slippage of the beam with respect to the phase of the plasma wave. The energy gain in a laser-plasma accelerator can be limited by this dephasing between the accelerating field of the plasma wave and the phase position of an electron beam in the wave. Dephasing will be the most severe limitation on energy gain for beams interacting with plasma waves driven by weakly-relativistic $a^{2}<1$ lasers in a matched plasma channel. Tapering of the plasma density can mitigate dephasing of the beam with respect to the plasma wave. To further enhance the performance of laser-plasma accelerators, it is anticipated that the next generation of experiments will employ plasma density tapering.

Analytic expressions were derived for the optimal taper to lock the phase of a relativistic beam in the longitudinal wake, allowing enhanced energy gains. Expressions for the maximum taper length over which the plasma density becomes singular, and the optimal laser pulse duration for maximizing the energy gain in the tapered plasma channel were also derived.

In a plasma channel, the phase velocities of the longitudinal and transverse wakefields differ, and control of the focusing fields in a plasma wave will require tapering to maintain the location of the beam in the phase of the transverse wake (i.e., focusing fields). An expression for the taper to phase lock the beam to the transverse wake was derived. With tapering to phase-lock the transverse wake, slippage in the phase of the longitudinal field results in a modest decrease in energy gain compared to the case of tapering to lock the phase of the longitudinal wake. It was shown that it is possible to lock the phases of both the longitudinal and transverse wakes by varying both the axial plasma density and the plasma channel radius, resulting in a varying laser spot size.

A discontinuous plasma density taper was considered to move the beam to plasma wave buckets closer to the drive laser, while maintaining a constant phase in the plasma wave. This technique can extend the interaction length and increase the energy gain. Although injecting a beam into a plasma wave bucket farther behind the laser yields comparable energy gains, over comparable distances. It was also shown that significant energy gains can be achieved by employing a linear plasma density taper, which could be simpler to realize experimentally. The slope for achieving the maximum energy gain was determined.
It should be noted that the laser pulse was assumed to be non-evolving, i.e., laser depletion, steepening, and self-focusing were neglected. The scale length for laser depletion and steepening is the pump depletion length $L_{p d} \sim\left(k_{0}^{2} / k_{p}^{3}\right) a^{-2}$, which for $a \ll 1$ is much longer than the tapering scale length $\sim k_{0}^{2} / k_{p}^{3}$. This assumption is hence only valid for weakly-relativistic laser intensities. Neglecting laser self-focusing requires $P / P_{c}<1$. For ultra-intense laser pulses, such that $a^{2} \gg 1$, the pulse evolution will modify the phase velocity of the plasma wave since the nonlinear plasma wavelength is a strong function of laser intensity in this regime. Tapering in the nonlinear laser intensity regime will be a topic of further study.

\section{ACKNOWLEDGMENTS}

The authors acknowledge fruitful discussions with J. Osterhoff, S. Hooker, B. Shadwick, and C. Geddes. This work was supported by the Director, Office of Science, Office of High Energy Physics, of the U.S. Department of Energy under Contract No. DE-AC0205CH11231, and by the Deutsche Forschungsgemeinschaft (DFG) through Transregio TR18 and supported by the DFG Cluster-of-Excellence "Munich-Centre for Advanced Photonics (MAP)".

\section{Appendix A: Laser evolution in a tapered plasma channel}

Applying the paraxial approximation, the linear evolution of the normalized laser intensity envelope in a parabolic (tapered) plasma channel is described by

$$
\left(\nabla_{\perp}^{2}+2 \mathrm{i} k_{0} \frac{\partial}{\partial z}\right) a=k_{p 0}^{2}(z)\left[1+\frac{r^{2}}{R_{\mathrm{ch}}^{2}(z)}\right] a,
$$

where an underdense plasma has been assumed such that $\gamma_{g} \gg 1$. Here the axial plasma density $k_{p 0}(z)$ and the channel radius $R_{\mathrm{ch}}(z)$ may vary due to the taper. Equation (A1) neglects relativistic self-guiding and plasma wave guiding, which will be valid for laser powers less than the critical power and weakly-relativistic laser intensities $a^{2} \ll 1$. The lowest-order Gaussian mode solution to Eq. (A1) can be expressed as

$$
a(z, r)=\frac{a_{0} r_{0}}{r_{s}(z)} \exp \left\{-[1-\mathrm{i} \alpha(z)] r^{2} / r_{s}^{2}(z)+\mathrm{i} \theta(z)\right\}
$$

where $r_{s}$ is the laser spot size, $\alpha$ is the phase front curvature, and $\theta$ is the phase shift. The evolution equation for the spot size $r_{s}$ is

$$
\frac{\mathrm{d}^{2} r_{s}}{\mathrm{~d} \tilde{z}^{2}}=\frac{r_{0}^{4}}{r_{s}^{3}}\left(1-\frac{k_{p 0}^{2} r_{s}^{4}}{4 R_{\mathrm{ch}}^{2}}\right)
$$

with the phase-front curvature is given by $\alpha=$ $\left(r_{s} / r_{0}\right) d\left(r_{s} / r_{0}\right) / d \tilde{z}$, and the phase shift evolution by 
$d \theta / d \tilde{z}=1-r_{0}^{2} / r_{s}^{2}$. Here $\tilde{z}=z / Z_{R}$ is the propagation distance normalized to the Rayleigh range, and the initial conditions $r_{s}(0)=r_{0}, d r_{s}(0) / d z=0, \theta(0)=0$, and $\alpha(0)=0$ were assumed (at the laser waist). Note that changes in the group velocity in the underdense plasma owing to the taper are neglected and $\beta_{g} \simeq 1$ is assumed. The changes in group velocity due to the taper ${ }^{21}$ yield terms that are of the order $\mathcal{O}\left(k_{p}^{4} / k_{0}^{4}\right)$, and, hence may be neglected.

As indicated by Eq. (A3) the condition for a matched laser pulse in a plasma channel is

$$
R_{\mathrm{ch}}(z)=k_{p 0}(z) r_{s}^{2}(z) / 2,
$$

and a matched laser pulse $r_{s}(z)=r_{0}=$ constant requires the channel radius to grow proportionally to the plasma wavenumber: $R_{\mathrm{ch}}(z)=k_{p 0}(z) r_{0}^{2} / 2$. This condition is equivalent to a constant channel depth, where the channel depth (change in plasma density at a laser spot size) normalized to the critical channel depth for guiding $\Delta n_{c}=m c^{2} / \pi \mathrm{e}^{2} r_{0}^{2}$ is $\Delta n / \Delta n_{c}=k_{p 0}^{2} r_{0}^{4} / 4 R_{\mathrm{ch}}^{2}$.

\section{Appendix B: Tapering in a narrow plasma channel}

In this Appendix, the plasma taper required for axial phase locking to order $\mathcal{O}\left(\kappa^{4}\right)$ in the transverse parameter $\kappa=2 /\left(k_{p 00} r_{0}\right)<1$ is calculated. To order $\mathcal{O}\left(\kappa^{4}\right)$, solutions to Eq. (10) yield a curvature term to the phase of the accelerating field. ${ }^{19}$ For a particle near the axis, the phase of the accelerating field is given by

$$
\psi_{z}=k_{p 0} \xi-\frac{2 \xi}{k_{p 0} R_{\mathrm{ch}}^{2}}=k_{p 0} \xi\left(1-\frac{\kappa^{4}}{2 \hat{k}_{p}^{4}}\right),
$$

where the matched laser condition is assumed $R_{\mathrm{ch}}=$ $k_{p 0} r_{0}^{2} / 2$. From Eq. (26) maintaining a highly-relativistic beam $\beta_{b} \simeq 1$ at a constant phase requires, to order $\mathcal{O}\left(\kappa^{4}\right)$,

$$
2\left|\psi_{0}\right| \frac{\mathrm{d} \hat{k}_{p}}{\mathrm{~d} \hat{z}}=\left(\hat{k}_{p}^{4}+\hat{k}_{p}^{2} \kappa^{2}-5 \kappa^{4} / 2\right) .
$$

Equation (B2) can be integrated to yield

$$
\frac{\hat{z}}{2\left|\psi_{0}\right|}=\frac{1}{3}\left(1-\hat{k}_{p}^{-3}\right)-\frac{\kappa^{2}}{5}\left(1-\hat{k}_{p}^{-5}\right)+\frac{\kappa^{4}}{2}\left(1-\hat{k}_{p}^{-7}\right),
$$

with the plasma density singularity reached at $\hat{z}_{s}=$ $\left(2\left|\psi_{0}\right| / 3\right)\left(1-3 \kappa^{2} / 5+3 \kappa^{4} / 2\right)$.

${ }^{1}$ E. Esarey, C. B. Schroeder, and W. P. Leemans, Rev. Mod. Phys. 81, 1229 (2009).

${ }^{2}$ F. Grüner, S. Becker, U. Schramm, T. Eichner, M. Fuchs, R. Weingartner, D. Habs, J. Meyer-ter-Vehn, M. Geissler, M. Ferrario, L. Serafini, B. van der Geer, H. Backe, W. Lauth, and S. Reiche, Appl. Phys. B 86, 431 (2007).

${ }^{3}$ H.-P. Schlenvoigt, K. Haupt, A. Debus, F. Budde, O. Jäckel, S. Pfotenhauer, H. Schwoerer, E. Rohwer, J. G. Gallacher, E. Brunetti, R. P. Shanks, S. M. Wiggins, and D. A. Jaroszynski, Nature Phys. 4, 130 (2008).

${ }^{4}$ M. Fuchs, R. Weingartner, A. Popp, Zs. Major, S. Becker, J. Osterhoff, I. Cortrie, R. Hörlein, G. D. Tsakiris, U. Schramm, T. P. Rowlands-Rees, S. M. Hooker, D. Habs, F. Krausz, S. Karsch, and F. Grüner, Nature Phys. 5, 826 (2009).

${ }^{5}$ W. Leemans and E. Esarey, Physics Today 62, 44 (2009).

${ }^{6}$ C. B. Schroeder, E. Esarey, C. G. R. Geddes, C. Tóth, and W. P. Leemans, Design considerations for a laser-plasma linear collider, in Advanced Accelerator Concepts, edited by C. B. Schroeder, E. Esarey, and W. Leemans, volume 1086, pp. 208-214, New York, 2009, AIP.

${ }^{7}$ W. P. Leemans, B. Nagler, A. J. Gonsalves, C. Tóth, K. Nakamura, C. G. R. Geddes, E. Esarey, C. B. Schroeder, and S. M. Hooker, Nature Phys. 2, 696 (2006).

${ }^{8}$ K. Nakamura, B. Nagler, C. Tóth, C. G. R. Geddes, C. B. Schroeder, E. Esarey, W. P. Leemans, A. J. Gonsalves, and S. M. Hooker, Phys. Plasmas 14, 056708 (2007).

${ }^{9}$ S. Karsch, J. Osterhoff, A. Popp, T. P. Rowlands-Rees, Z. Major, M. Fuchs, B. Marx, R. Hörlein, K. Schmid, L. Veisz, S. Becker, U. Schramm, B. Hidding, G. Pretzler, D. Habs, F. Grüner, F. Krausz, and S. M. Hooker, New J. Phys. 9, 415 (2007).

${ }^{10}$ E. Esarey, P. Sprangle, J. Krall, and A. Ting, IEEE J. Quantum Electron. 33, 1879 (1997).

${ }^{11}$ C. G. R. Geddes, C. Toth, J. van Tilborg, E. Esarey, C. B. Schroeder, J. Cary, and W. P. Leemans, Phys. Rev. Lett. 95, 145002 (2005).

${ }^{12}$ E. Esarey and M. Pilloff, Phys. Plasmas 2, 1432 (1995).

${ }^{13}$ B. A. Shadwick, C. B. Schroeder, and E. Esarey, Phys. Plasmas 16 (2009).

${ }^{14}$ T. Katsouleas, Phys. Rev. A 33, 2056 (1986).

${ }^{15}$ S. V. Bulanov, V. A. Vshivkov, G. I. Dudnikova, N. M. Naumova, F. Pegoraro, and I. V. Pogorelsky, Plasma Phys. Rep. 23, 259 (1997).

${ }^{16}$ A. Pukhov and I. Kostyukov, Phys. Rev. E 77, 025401 (2008).

${ }^{17}$ P. Sprangle, B. Hafizi, J. R. Peñano, R. F. Hubbard, A. Ting, C. I. Moore, D. F. Gordon, A. Zigler, D. Kaganovich, and T. M. Antonsen, Jr., Phys. Rev. E 63, 056405 (2001).

${ }^{18}$ P. Sprangle, E. Esarey, J. Krall, and G. Joyce, Phys. Rev. Lett. 69, 2200 (1992).

${ }^{19}$ N. E. Andreev, L. M. Gorbunov, V. I. Kirsanov, K. Nakajima, and A. Ogata, Phys. Plasmas 4, 1145 (1997).

${ }^{20}$ E. Cormier-Michel, E. Esarey, C. G. R. Geddes, C. B. Schroeder, K. Paul, J. P. Mullowney, J. Cary, and W. P. Leemans, "Control of focusing fields in laser plasma accelerators using higher order modes", Phys. Rev. E, submitted for publication.

${ }^{21}$ P. Jha, R. G. Singh, A. K. Upadhyaya, and R. K. Mishra, Phys. Plasmas 15, 033101 (2008). 